DESY 08-096

arXiv:0807.2405

\title{
Threshold resummation for squark-antisquark and gluino-pair production at the LHC
}

\author{
A. Kulesza ${ }^{a}$ AND L. MOTYKA ${ }^{b, c} \dagger$ \\ ${ }^{a}$ Deutsches Elektronen-Synchrotron DESY, Notkestrasse 85, \\ D-22607 Hamburg, Germany \\ ${ }^{b}$ II Institute for Theoretical Physics, University of Hamburg, \\ Luruper Chaussee 149, D-22761, Germany \\ ${ }^{c}$ Institute of Physics, Jagellonian University, Reymonta 4, \\ 30-059 Kraków, Poland
}

July 15, 2008

\begin{abstract}
We study the effect of soft gluon emission in the hadroproduction of squark-antisquark and gluino-gluino pairs at the next-to-leading logarithmic (NLL) accuracy within the framework of the minimal supersymmetric model. The one-loop soft anomalous dimension matrices controlling the colour evolution of the underlying hard-scattering processes are calculated. We present the resummed total cross sections and show numerical results for proton-proton collisions at $14 \mathrm{TeV}$. The size of the NLL contribution to the cross section and the reduction of the scale dependence of the theoretical predictions due to including soft gluon effects are discussed.
\end{abstract}

\footnotetext{
†anna.kulesza@desy.de, leszek.motyka@desy.de
} 


\section{Introduction}

The search for new physics phenomena at the $\mathrm{TeV}$ scale begins at the Large Hadron Collider (LHC) this year. Among the proposed models of new physics, extentions of the Standard Model (SM) involving supersymmetry (SUSY) are one of the best motivated. Over the years much attention has been focused on the Minimal Supersymmetric Standard Model (MSSM) [1], characterized by the minimal content of supersymmetric particles and $R$-parity conservation. In the MSSM the SUSY partners of the SM particles are always produced in pairs.

The dominant production processes of sparticles at the LHC are those involving pairs of coloured particles, i.e. squarks and gluinos, in the final state [2]. The discovery of squarks and gluinos should be possible for masses of up to $2 \mathrm{TeV}$ [3]. Depending on the outcome of the experimental searches, predictions for the total rates for these production processes will either help to determine the masses of the sparticles [4] or to draw exclusion limits for the mass parameters. It is therefore important to know the LHC cross sections for production of squarks and gluinos with high theoretical accuracy. In particular, control over effects of higher-order radiative corrections leads to a stabilization of the theoretical predictions w.r.t. variation of the renormalisation and factorisation scales and, consequently, to a smaller theoretical error.

The leading-order ( $\mathrm{LO}$ ) total cross sections and the corresponding next-to-leading order (NLO) SUSY-QCD corrections are known for all hadroproduction processes of pairs of squarks and gluinos [5, 6, 7]. They have been found to be positive and large, especially for the gluino-pair production. As pointed out in [7], an important part of the contributions to the hadronic cross sections comes from the energy region near the partonic production threshold. The threshold region is reached when the square of the partonic center-of-mass (c.o.m.) energy, $\hat{s}$, approaches $4 \mathrm{~m}^{2}$, where $m$ is the average particle mass in the produced pair. The velocity of the produced heavy particles in the partonic c.o.m. system $\beta \equiv \sqrt{1-4 m^{2} / \hat{s}}$ is then small, $\beta \ll 1$. In this region, two types of corrections dominate: Coulomb corrections, due to exchange of gluons between slowly moving massive particles, and soft gluon corrections, due to emission of low energy gluons off the coloured initial and final states. The large size of the soft gluon emission contributions can be traced down, for the perturbative $n$-th order correction, to the logarithmic terms of the form $\alpha_{\mathrm{s}}^{n} \log ^{k}\left(\beta^{2}\right)$ where $k=2 n, \ldots, 0$. The effects of the soft gluon emission can be taken into account to all orders in perturbation theory by performing resummation of the threshold logarithms. Resummed predictions are particularly important for processes with large masses in the final states, since then the bulk of the production comes from the threshold region. This is exactly the case for the production of the sparticles which are expected to be heavier than the SM particles. Additionally, if the partonic subprocesses involve gluons in the initial state, the soft gluon effects, and thus the impact of resummation, are expected to be significant due to the high colour charge of the gluons. 
In this letter, we report on the calculation of threshold-resummed cross sections for hadroproduction of gluino-gluino ( $\tilde{g} \tilde{g})$ and squark-antisquark $(\tilde{q} \overline{\tilde{q}})$ pairs. Among the pair-production processes of coloured sparticles at the LHC, the $\tilde{g} \tilde{g}$ production receives the largest NLO SUSY-QCD correction [7], and the NLO $K$-factor, $K_{\mathrm{NLO}}$, can reach 2 for gluino mass $m_{\tilde{g}}=1 \mathrm{TeV}$. The corrections to the $\tilde{q} \overline{\tilde{q}}$ total cross section can be also sizeable $K_{\mathrm{NLO}} \simeq 1.3$ for the squark mass, $m_{\tilde{q}}=1 \mathrm{TeV}$, and are the second largest in a certain range of mass parameters. The hadronic production of $\tilde{g} \tilde{g}$ and $\tilde{q} \overline{\tilde{q}}$ pairs are scattering processes with a non-trivial colour flow structure. At the level of next-to-leading logarithms (NLL), resummation requires including contributions from soft gluons emitted at wide angles. Such emission is sensitive to the colour flow of the underlying hard scattering and the evolution of the colour exchange is governed by the one-loop soft anomalous dimension matrix [8, 9, 10, 11, 12. These matrices have been calculated for heavy-quark and dijet production [9, 13, 8, 11] and the general results for any $2 \rightarrow n$ QCD process with massless particles in the final state have been derived at one- [12], and two-loops [14]. Recently, the two-loop anomalous dimension for the pair production of heavy quarks has been also determined in the threshold limit [15. Here we present the explicit form of the soft anomalous dimension matrices for partonic subprocesses contributing to $\tilde{g} \tilde{g}$ hadroproduction and apply the resummation formalism at the NLL level to evaluate the correction due to soft gluon emission for the production of $\tilde{g} \tilde{g}$ and $\tilde{q} \overline{\tilde{q}}$ pairs at the LHC.

\section{Soft gluon resummation}

In this letter we consider the hadronic production of gluino-pair and squark-antisquark final states, $h_{1} h_{2} \rightarrow \tilde{g} \tilde{g}$ and $h_{1} h_{2} \rightarrow \tilde{q} \overline{\tilde{q}}$, where we sum over the left- and right- chiralities of squarks and over the spins of gluinos. At LO, these two processes receive contributions from the following partonic channels:

$$
q \bar{q} \rightarrow \tilde{g} \tilde{g}, \quad \bar{q} q \rightarrow \tilde{g} \tilde{g}, \quad g g \rightarrow \tilde{g} \tilde{g}
$$

and

$$
q \bar{q} \rightarrow \tilde{q} \overline{\tilde{q}}, \quad \bar{q} q \rightarrow \tilde{q} \overline{\tilde{q}}, \quad g g \rightarrow \tilde{q} \overline{\tilde{q}},
$$

respectively. The resummation of the soft gluon corrections is carried out in Mellin- $N$ space in the variable $\rho=4 \mathrm{~m}^{2} / S$ with $S$ being the square of the hadronic c.o.m energy and $m$ the mass of the final-state particle. In the Mellin space, the moments of the partonic cross section $i j \rightarrow k l$ are given by

$$
\hat{\sigma}_{i j \rightarrow k l, N}\left(m^{2}, \mu_{F}^{2}, \mu_{R}^{2}\right) \equiv \int_{0}^{1} d \hat{\rho} \hat{\rho}^{N-1} \hat{\sigma}_{i j \rightarrow k l}\left(\hat{\rho}, m^{2}, \mu_{F}^{2}, \mu_{R}^{2}\right)
$$

with $\hat{\rho}=4 m^{2} / \hat{s}$.

The evolution of colour exchange in non-collinear soft gluon emission, which has to be taken into account at the NLL accuracy, is governed by the soft anomalous dimension matrix $\Gamma^{i j \rightarrow k l}[8$, 
9, 10, 11, 12. In an orthogonal basis in the colour space for which the matrix $\Gamma^{i j \rightarrow k l}$ is diagonal, the NLL resummed cross section in the $N$-space has the form [9, 13]

$$
\hat{\sigma}_{i j \rightarrow k l, N}^{(\mathrm{res})}=\sum_{I} \hat{\sigma}_{i j \rightarrow k l, I, N}^{(0)} \widetilde{C}_{i j \rightarrow k l, I} \Delta_{N+1}^{i} \Delta_{N+1}^{j} \Delta_{i j \rightarrow k l, I, N+1}^{(\mathrm{int})},
$$

where we suppress explicit dependence on the scales. The index $I$ in Eq. (2) distinguishes between contributions from different colour channels. The colour-channel-dependent contributions to the LO partonic cross sections in Mellin-moment space are denoted by $\hat{\sigma}_{i j \rightarrow k l, I, N}^{(0)}$ and will be presented elsewhere [16]. The radiative factors $\Delta_{N}^{i}$ describe the effect of the soft gluon radiation collinear to the initial state partons and are universal. Large-angle soft gluon emission is accounted for by the factors $\Delta_{i j \rightarrow k l, I, N}^{(i n t)}$ which depend on the partonic process under consideration and the colour configuration of the participating particles. The functions $\widetilde{C}_{i j \rightarrow k l, I}$ are in general obtained by comparison of the resummed formulas with the fixed-order expressions. They consist of Coulomb corrections and $N$-independent terms (often referred to in the literature under the common symbol $C_{i j \rightarrow k l}$ ), containing hard contributions from virtual corrections. For the most complete treatment of the threshold effects, the Coulomb corrections should be resummed, see e.g. [17, 13. The concurrent treatment of the Coulomb and soft gluon corrections is, however, beyond the scope of the work reported on here.

The expressions for the radiative factors in the $\overline{\mathrm{MS}}$ factorisation scheme read (see e.g. [13])

$$
\begin{aligned}
\ln \Delta_{N}^{i} & =\int_{0}^{1} d z \frac{z^{N-1}-1}{1-z} \int_{\mu_{F}^{2}}^{4 m^{2}(1-z)^{2}} \frac{d q^{2}}{q^{2}} A_{i}\left(\alpha_{\mathrm{S}}\left(q^{2}\right)\right), \\
\ln \Delta_{i j \rightarrow k l, I, N}^{\text {(int) }} & =\int_{0}^{1} d z \frac{z^{N-1}-1}{1-z} D_{i j \rightarrow k l, I}\left(\alpha_{\mathrm{S}}\left(4 m^{2}(1-z)^{2}\right)\right) .
\end{aligned}
$$

The coefficients $\mathcal{F}=A_{i}, D_{i j \rightarrow k l, I}$ are power series in the coupling constant $\alpha_{\mathrm{s}}, \mathcal{F}=\left(\frac{\alpha_{\mathrm{s}}}{\pi}\right) \mathcal{F}^{(1)}+$ $\left(\frac{\alpha_{\mathrm{s}}}{\pi}\right)^{2} \mathcal{F}^{(2)}+\ldots$ The universal LL and NLL coefficients $A_{i}^{(1)}, A_{i}^{(2)}$ are well known [18, 19] and given by

$$
\begin{aligned}
& A_{i}^{(1)}=C_{i}, \\
& A_{i}^{(2)}=\frac{1}{2} C_{i}\left(\left(\frac{67}{18}-\frac{\pi^{2}}{6}\right) C_{\mathrm{A}}-\frac{5}{9} n_{\mathrm{f}}\right)
\end{aligned}
$$

with $C_{g}=C_{\mathrm{A}}=3$, and $C_{q}=C_{\mathrm{F}}=4 / 3$. The customary NLL expansions of the radiative factors can be found in [13]. Since after the expansion of the exponentials the terms constant in $N$ contained in $C_{i j \rightarrow k l}$ generate contributions of the next-to-next-to-leading logarithmic (NNLL) order, and we do not include Coulomb corrections here, we keep $\widetilde{C}_{i j \rightarrow k l, I}=1$ for the purpose of the calculations.

The NLL coefficients $D_{i j \rightarrow \tilde{q} \overline{\tilde{q}}, I}^{(1)}$ are the same as in the heavy-quark production and were calculated in $[9,13$,

$$
\begin{aligned}
& D_{q \bar{q} \rightarrow \tilde{q} \overline{\tilde{q}}, 1}^{(1)}=D_{g g \rightarrow \tilde{q} \overline{\tilde{q}}, 1}^{(1)}=0, \\
& D_{q \bar{q} \rightarrow \tilde{q} \tilde{\tilde{q}}, 2}^{(1)}=D_{g g \rightarrow \tilde{q} \overline{\tilde{q}}, 2}^{(1)}=-C_{\mathrm{A}},
\end{aligned}
$$


with the index $I=1,2$ corresponding to the singlet and octet $\{\mathbf{1}, \mathbf{8}\}$ exchange in the $s$-channel. For the $\tilde{g} \tilde{g}$ production we first study the soft anomalous dimension matrices $\Gamma^{i j \rightarrow \tilde{g} \tilde{g}}$ and then derive the coefficients $D_{i j \rightarrow \tilde{g} \tilde{g}, I}^{(1)}$. Apart from obvious colour-dependent modifications, the matrices $\Gamma^{i j \rightarrow \tilde{g} \tilde{g}}$ are defined in the same way as the soft anomalous dimension matrices for the heavyquark production process in [9]. For the purpose of this calculation we consider the partonic processes

$$
q\left(p_{1}, \alpha_{1}\right) \bar{q}\left(p_{2}, \alpha_{2}\right) \rightarrow \tilde{g}\left(p_{3}, a_{3}\right) \tilde{g}\left(p_{4}, a_{4}\right)
$$

and

$$
g\left(p_{1}, a_{1}\right) g\left(p_{2}, a_{2}\right) \rightarrow \tilde{g}\left(p_{3}, a_{3}\right) \tilde{g}\left(p_{4}, a_{4}\right),
$$

where $p_{i}$ are the particle four-momenta and $\alpha_{i}$ and $a_{i}$ are the colour indices in the fundamental and adjoint representation of $\mathrm{SU}(3)$, respectively.

Three independent tensors are forming the basis in the space of colour exchanges for the $q \bar{q} \rightarrow \tilde{g} \tilde{g}$ process. We choose an orthogonal $s$-channel basis $\left\{c_{I}^{q}\right\}, I=1,2,3$ :

$$
c_{1}^{q}=\delta^{\alpha_{1} \alpha_{2}} \delta^{a_{3} a_{4}}, \quad c_{2}^{q}=T_{\alpha_{2} \alpha_{1}}^{b} d^{b a_{3} a_{4}}, \quad c_{3}^{q}=i T_{\alpha_{2} \alpha_{1}}^{b} f^{b a_{3} a_{4}},
$$

where $T^{b}$ matrices are the $\mathrm{SU}(3)$ generators, and the tensors correspond to $\mathbf{1}, \mathbf{8}_{\mathbf{S}}$ and $\mathbf{8}_{\mathbf{A}}$ representations, respectively. For the $g g$ channel there are eight independent colour tensors. Following [11] we choose an orthogonal basis $\left\{c_{I}^{g}\right\}(I=1,2, \ldots, 8)$ consisting of five tensors $c_{1}^{g}$, $c_{2}^{g}, c_{3}^{g}, c_{4}^{g}, c_{5}^{g}$ corresponding to the $\mathbf{1}, \mathbf{8}_{\mathbf{S}}, \mathbf{8}_{\mathbf{A}}, \mathbf{1 0} \oplus \overline{\mathbf{1 0}}, \mathbf{2 7}$ representations in the $s$-channel, and three additional tensors $c_{6}^{g}, c_{7}^{g}, c_{8}^{g}$. The base tensors are

$$
\begin{aligned}
c_{1}^{g} & =\frac{1}{8} \delta^{a_{1} a_{2}} \delta^{a_{3} a_{4}}, \\
c_{2}^{g} & =\frac{3}{5} d^{a_{1} a_{2} b} d^{b a_{3} a_{4}}, \\
c_{3}^{g} & =\frac{1}{3} f^{a_{1} a_{2} b} f^{b a_{3} a_{4}}, \\
c_{4}^{g} & =\frac{1}{2}\left(\delta^{a_{1} a_{3}} \delta^{a_{2} a_{4}}-\delta^{a_{1} a_{4}} \delta^{a_{2} a_{3}}\right)-\frac{1}{3} f^{a_{1} a_{2} b} f^{b a_{3} a_{4}}, \\
c_{5}^{g} & =\frac{1}{2}\left(\delta^{a_{1} a_{3}} \delta^{a_{2} a_{4}}+\delta^{a_{1} a_{4}} \delta^{a_{2} a_{3}}\right)-\frac{1}{8} \delta^{a_{1} a_{2}} \delta^{a_{3} a_{4}}-\frac{3}{5} d^{a_{1} a_{2} b} d^{b a_{3} a_{4}}, \\
c_{6}^{g} & =\frac{i}{4}\left(f^{a_{1} a_{2} b} d^{b a_{3} a_{4}}+d^{a_{1} a_{2} b} f^{b a_{3} a_{4}}\right), \\
c_{7}^{g} & =\frac{i}{4}\left(f^{a_{1} a_{2} b} d^{b a_{3} a_{4}}-d^{a_{1} a_{2} b} f^{b a_{3} a_{4}}\right), \\
c_{8}^{g} & =\frac{i}{4}\left(d^{a_{1} a_{3} b} f^{b a_{2} a_{4}}+f^{a_{1} a_{3} b} d^{b a_{2} a_{4}}\right) .
\end{aligned}
$$

We introduce the notation

$$
\begin{aligned}
\bar{T} & \equiv \ln \left(\frac{m^{2}-\hat{t}}{\sqrt{m^{2} \hat{s}}}\right)-\frac{1-i \pi}{2} \\
\bar{U} & \equiv \ln \left(\frac{m^{2}-\hat{u}}{\sqrt{m^{2} \hat{s}}}\right)-\frac{1-i \pi}{2} \\
\bar{S} & \equiv-\frac{L_{\beta}+1}{2}
\end{aligned}
$$


where the Mandelstam variables are given by

$$
\hat{s}=\left(p_{1}+p_{2}\right)^{2}, \quad \hat{t}=\left(p_{1}-p_{3}\right)^{2}, \quad \hat{u}=\left(p_{1}-p_{4}\right)^{2},
$$

and $L_{\beta}=\frac{1}{\beta}\left(1-2 m^{2} / \hat{s}\right)\left(\ln \frac{1-\beta}{1+\beta}+i \pi\right)$. We also define $\Lambda \equiv \bar{T}+\bar{U}, \Omega \equiv \bar{T}-\bar{U}$.

Using the results for the one-loop eikonal integrals from [9] we calculate the matrices $\Gamma^{i j \rightarrow \tilde{g} \tilde{g}}[16]$. For the $q \bar{q}$ channel we obtain

$$
\Gamma^{q \bar{q} \rightarrow \tilde{g} \tilde{g}}=\frac{\alpha_{s}}{\pi}\left[\left(\begin{array}{ccc}
6 \bar{S} & 0 & -\Omega \\
0 & 3 \bar{S}+\frac{3}{2} \Lambda & -\frac{3}{2} \Omega \\
-2 \Omega & -\frac{5}{6} \Omega & 3 \bar{S}+\frac{3}{2} \Lambda
\end{array}\right)-\frac{4}{3} i \pi \hat{\mathbf{I}}\right] .
$$

The matrix for the $g g$ channel has the block form

$$
\Gamma^{g g \rightarrow \tilde{g} \tilde{g}}=\frac{\alpha_{s}}{\pi}\left[\left(\begin{array}{cc}
\Gamma_{5} & \hat{\mathbf{0}} \\
\hat{\mathbf{0}} & \Gamma_{3}
\end{array}\right)-3 i \pi \hat{\mathbf{I}}\right]
$$

where the five-dimensional matrix reads

$$
\Gamma_{5}=\left(\begin{array}{ccccc}
6 \bar{S} & 0 & 6 \Omega & 0 & 0 \\
0 & 3 \bar{S}+\frac{3}{2} \Lambda & \frac{3}{2} \Omega & 3 \Omega & 0 \\
\frac{3}{4} \Omega & \frac{3}{2} \Omega & 3 \bar{S}+\frac{3}{2} \Lambda & 0 & \frac{9}{4} \Omega \\
0 & \frac{6}{5} \Omega & 0 & 3 \Lambda & \frac{9}{5} \Omega \\
0 & 0 & \frac{2}{3} \Omega & \frac{4}{3} \Omega & 4 \Lambda-2 \bar{S}
\end{array}\right)
$$

and the three-dimensional matrix $\Gamma_{3}$ is diagonal,

$$
\Gamma_{3}=\operatorname{diag}(3(\bar{S}+\bar{U}), 3(\bar{S}+\bar{T}), 3(\bar{T}+\bar{U})) .
$$

As in [9], the matrices $\Gamma$ contain terms from the one-loop eikonal integrals for $2 \rightarrow 2$ scattering with massive particles in the final state and are shifted by half of the soft anomalous dimension for the Drell-Yan cross section 1]. The matrices (9), (10) complement the results of [11] for the case of pair-production of massive colour-octet particles with equal masses.

At the threshold, $\hat{s} \rightarrow 4 m^{2}$, the soft anomalous dimension matrices approach the diagonal form,

$$
\begin{aligned}
& \Gamma^{q \bar{q} \rightarrow \tilde{g} \tilde{g}} \rightarrow \frac{\alpha_{s}}{\pi} \operatorname{diag}\left(\gamma_{1}^{g}, \gamma_{2}^{g}, \ldots, \gamma_{8}^{g}\right) \\
& \Gamma^{q \bar{q} \rightarrow \tilde{q} \tilde{q}} \rightarrow \frac{\alpha_{s}}{\pi} \operatorname{diag}\left(\gamma_{1}^{q}, \gamma_{2}^{q}, \gamma_{3}^{q}\right) .
\end{aligned}
$$

\footnotetext{
${ }^{1}$ Gauge dependence in the one-loop integrals cancels against gauge dependence of the incoming-jet factors (which corresponds to gauge dependence of the soft function in the resummed Drell-Yan cross section 11, 23]), leaving the results presented here gauge-invariant.
} 
The off-diagonal terms, proportional to $\Omega$, vanish like $\beta$ for $\beta \rightarrow 0$ and may be neglected. Using $D_{g g \rightarrow \tilde{g} \tilde{g}, I}^{(1)}=2 \operatorname{Re}\left(\gamma_{I}^{g}\right)$ and $D_{q \bar{q} \rightarrow \tilde{g} \tilde{g}, I}^{(1)}=2 \operatorname{Re}\left(\gamma_{I}^{q}\right)[10$, 11] we obtain

$$
\begin{aligned}
& \left\{D_{g g \rightarrow \tilde{g} \tilde{g}, I}^{(1)}\right\}=\{0,-3,-3,-6,-8 ;-3,-3,-6\} \\
& \left\{D_{q \bar{q} \rightarrow \tilde{g} \tilde{g}, I}^{(1)}\right\}=\{0,-3,-3\} .
\end{aligned}
$$

Note that the values of the $D^{(1)}$-coefficients are the negative values of the quadratic Casimir operators for the $\mathrm{SU}(3)$ representations for the outgoing state. This agrees with the physical picture of the soft gluon radiation from the total colour charge of the heavy-particle pair produced at threshold [13].

\section{Numerical results}

In the phenomenological analysis we consider a wide range of gluino and squark masses. Left- and right-handed squarks of all flavours are assumed to be mass degenerate. For the $\tilde{g} \tilde{g}$ production we vary 2 the gluino mass, $m_{\tilde{g}}$, between $200 \mathrm{GeV}$ and $2 \mathrm{TeV}$. Similarly, for the $\tilde{q} \overline{\tilde{q}}$ production we take $200 \mathrm{GeV}<m_{\tilde{q}}<2 \mathrm{TeV}$. We present the results for a fixed ratio of gluino and squark masses, $r=\frac{m_{\tilde{g}}}{m_{\tilde{q}}}$, and choose the following values $r=0.5,0.81 .21 .6,2.0$. The $\tilde{q} \overline{\tilde{q}}$ cross section accounts for production of all $\tilde{q} \overline{\tilde{q}}$ flavour combinations apart from the ones with scalar top particles.

The main phenomenological results of this letter are the resummation-improved predictions for the $p p \rightarrow \tilde{q} \overline{\tilde{q}}$ and $p p \rightarrow \tilde{g} \tilde{g}$ total cross sections at $\sqrt{S}=14 \mathrm{TeV}$. The resummation-improved cross sections are obtained through matching the NLL resummed expressions with the full NLO cross sections

$$
\begin{aligned}
\sigma_{h_{1} h_{2} \rightarrow k l}^{(\text {match })}\left(\rho, m^{2},\left\{\mu^{2}\right\}\right) & =\sum_{i, j=q, \bar{q}, g} \int_{C_{\mathrm{M} P}-i \infty}^{C_{\mathrm{M} P}+i \infty} \frac{d N}{2 \pi i} \rho^{-N} f_{i / h_{1}}^{(N+1)}\left(\mu_{F}^{2}\right) f_{j / h_{2}}^{(N+1)}\left(\mu_{F}^{2}\right) \\
& \times\left[\hat{\sigma}_{i j \rightarrow k l, N}^{(\mathrm{res})}\left(m^{2},\left\{\mu^{2}\right\}\right)-\left.\hat{\sigma}_{i j \rightarrow k l, N}^{(\mathrm{res})}\left(m^{2},\left\{\mu^{2}\right\}\right)\right|_{(\mathrm{NLO})}\right] \\
& +\sigma_{h_{1} h_{2} \rightarrow k l}^{(\mathrm{NLO})}\left(\rho, m^{2},\left\{\mu^{2}\right\}\right),
\end{aligned}
$$

where $\left\{\mu^{2}\right\}=\left\{\mu_{F}^{2}, \mu_{R}^{2}\right\}, \hat{\sigma}_{i j \rightarrow k l, N}^{\text {(res) }}$ is given in Eq. (2) and $\left.\hat{\sigma}_{i j \rightarrow k l, N}^{\text {(res) }}\right|_{(\mathrm{NLO})}$ represents its perturbative expansion truncated at NLO. The moments of the parton distribution functions $f_{i / h}\left(x, \mu_{F}^{2}\right)$ are defined in the standard way

$$
f_{i / h}^{(N)}\left(\mu_{F}^{2}\right) \equiv \int_{0}^{1} d x x^{N-1} f_{i / h}\left(x, \mu_{F}^{2}\right) .
$$

The inverse Mellin transform (15) is evaluated numerically using a contour in the complex- $N$ space according to the "Minimal Prescription" method developed in Ref. [20].

\footnotetext{
${ }^{2}$ For the highest masses considered here, the experimental exploration will require the integrated luminosity of $\mathcal{O}\left(100 \mathrm{fb}^{-1}\right)$.
} 

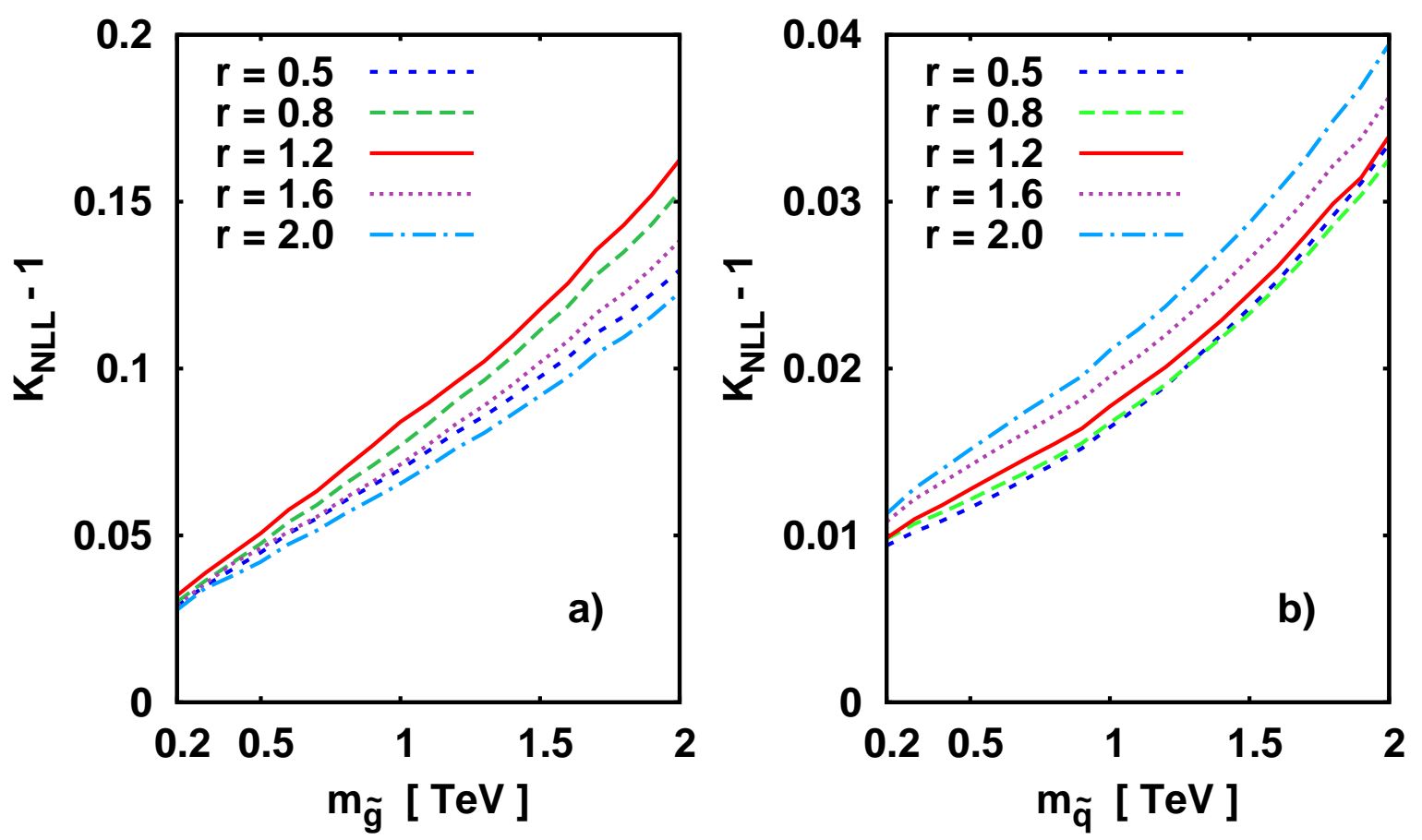

Figure 1: The relative NLL correction $K_{\mathrm{NLL}}-1$ for the $\tilde{g} \tilde{g}$ (a) and the $\tilde{q} \overline{\tilde{q}}$ (b) total production cross section at the LHC as a function of gluino and squark mass, respectively; $r=m_{\tilde{g}} / m_{\tilde{q}}$.

The NLO cross sections are evaluated using PROSPINo [21, the numerical package based on calculations employing the $\overline{\mathrm{MS}}$ renormalisation and factorisation schemes. We use the CTEQ6M [22] parameterization of parton distribution functions (pdfs) for all numerical predictions. Similarly to other available pdfs, the assumption of five massless quark flavours active at large scales is made in the CTEQ6M parameterization. Consequently, in the NLO and NLL calculations we use the two-loop $\overline{\mathrm{MS}}$ QCD running coupling constant $\alpha_{\mathrm{s}}$ with $n_{\mathrm{f}}=5$ and $\Lambda_{\mathrm{QCD}}^{(5)}=0.226 \mathrm{GeV}$. The effects due to virtual top quarks and virtual sparticles in the running of $\alpha_{\mathrm{s}}$ and in the evolution of pdfs are thus not included in our predictions. However, the value of the top mass, $m_{t}=175 \mathrm{GeV}$, enters the matched NLL cross sections through the NLO corrections.

All numerical calculations were performed using two independent computer codes. As a first check we reproduced numerically, and analytically, the LO results [7, 21]. We also verified that the NLL resummed expression in $N$-space generates the same logarithmic terms as these present in the Mellin transforms of the NLO correction in the threshold limit given in [7].

The relative corrections from soft gluon resummation to the $\tilde{g} \tilde{g}$ and $\tilde{q} \overline{\tilde{q}}$ NLO production cross sections at the LHC,

$$
K_{\mathrm{NLL}}-1 \equiv \sigma^{(\mathrm{match})} / \sigma^{(\mathrm{NLO})}-1
$$



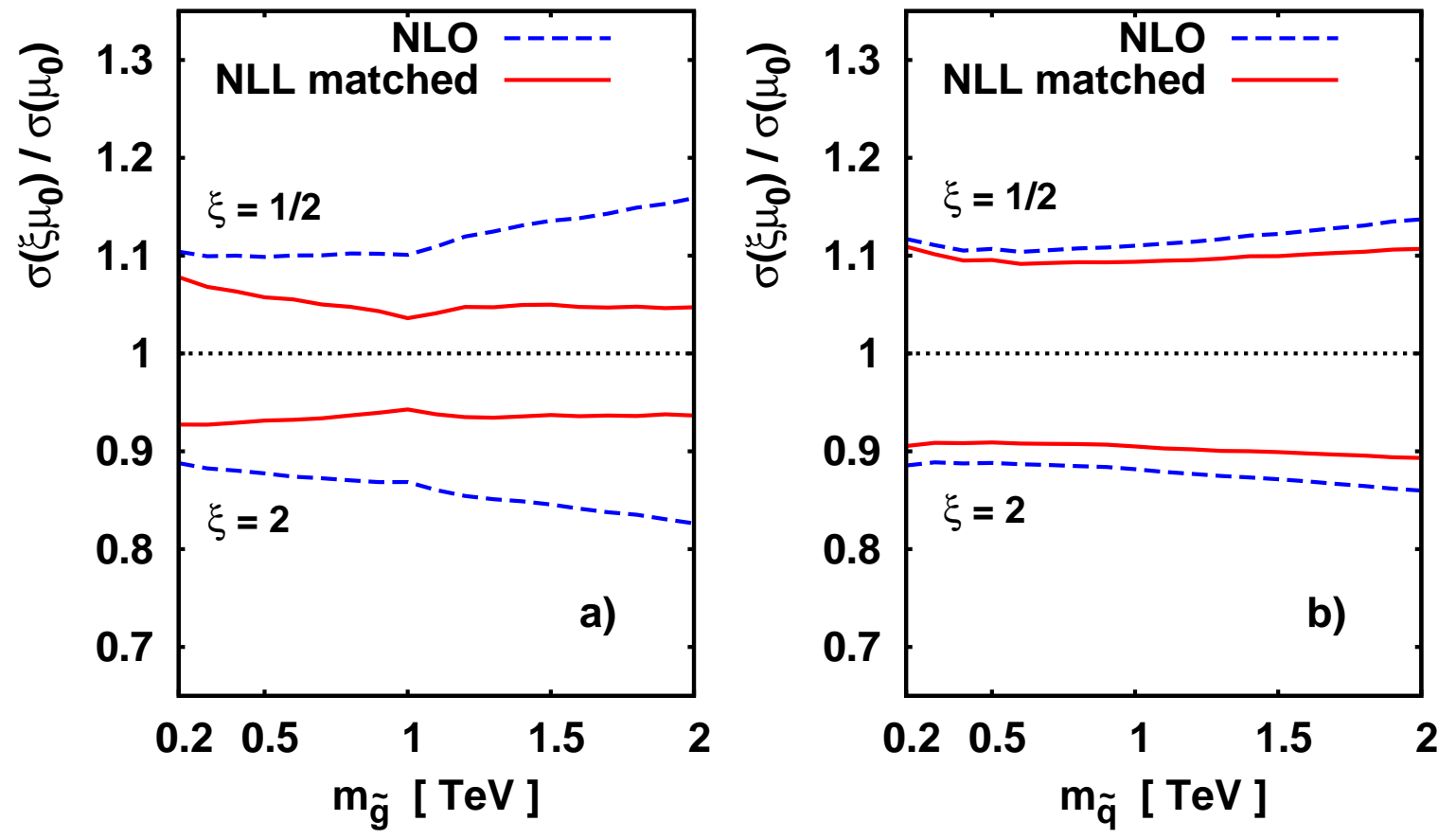

Figure 2: Scale dependence of the total $\tilde{g} \tilde{g}$ (a) and $\tilde{q} \overline{\tilde{q}}$ (b) production cross section at the LHC (see the text for explanation).

are presented in Fig. 1a and Fig. 1b, respectively. In the plots we set the scales $\mu_{F}=\mu_{R}=\mu_{0}$, where $\mu_{0}=m_{\tilde{g}}\left(\mu_{0}=m_{\tilde{q}}\right)$ for the $\tilde{g} \tilde{g}$ production (the $\tilde{q} \tilde{\tilde{q}}$ production). $K_{\text {NLL }}$ grows with the final-state mass and depends on the mass ratio $r$ in a moderate way. The correction, $K_{\mathrm{NLL}}-1$, reaches $16 \%(8 \%)$ for the $\tilde{g} \tilde{g}$ production with $r=1.2$ and $m_{\tilde{g}}=2 \mathrm{TeV}(1 \mathrm{TeV})$, and $4 \%(2 \%)$ for the $\tilde{q} \tilde{\tilde{q}}$ production with $r=2$ and $m_{\tilde{q}}=2 \mathrm{TeV}(1 \mathrm{TeV})$. The stronger effect found in the $\tilde{g} \tilde{g}$ production follows from the dominance of the $g g \rightarrow \tilde{g} \tilde{g}$ channel, and hence more intense soft gluon radiation.

We also investigate the dependence of the matched NLL cross section on the values of factorisation and renormalisation scales, in comparison to the NLO cross section. To illustrate our results we choose $\mu=\mu_{F}=\mu_{R}$ and $r=1$.2. In Fig. 2a and Fig. 2b we plot the ratios $\sigma^{\mathrm{NLO}}\left(\mu=\xi \mu_{0}\right) / \sigma^{\mathrm{NLO}}\left(\mu=\mu_{0}\right)$ and $\sigma^{(\text {match })}\left(\mu=\xi \mu_{0}\right) / \sigma^{(\text {match })}\left(\mu=\mu_{0}\right)$, obtained by varying $\xi$ between $\xi=1 / 2$ and $\xi=2$, for $\tilde{g} \tilde{g}\left(\mu_{0}=m_{\tilde{g}}\right)$ and $\tilde{q} \overline{\tilde{q}}\left(\mu_{0}=m_{\tilde{q}}\right)$ production, respectively. Due to resummation, the scale sensitivity of the $\tilde{g} \tilde{g}$ production cross section reduces significantly, by a factor of $\sim 3(\sim 2)$ at $m_{\tilde{g}}=2 \mathrm{TeV}\left(m_{\tilde{g}}=1 \mathrm{TeV}\right)$. At $m_{\tilde{g}}>1 \mathrm{TeV}$ the theoretical error of the matched NLL $\tilde{g} \tilde{g}$ cross section, defined by changing the scale $\mu=\mu_{F}=\mu_{R}$ around $\mu_{0}=m_{\tilde{g}}$ by a factor of 2 , is around $5 \%$. In the case of the $\tilde{q} \overline{\tilde{q}}$ production, the reduction of the scale dependence due to including soft gluon corrections in the theoretical predictions is moderate. 
Acknowledgments. We thank J. Bartels and G. Sterman for comments on the manuscript. L.M. is supported by the DFG grant no. SFB 676 .

\section{References}

[1] H. E. Haber and G. L. Kane, Phys. Rept. 117, 75 (1985).

[2] see e.g. M. Drell, R. Godbole and P. Roy, "Theory and Phenomenology of Sparticles", World Scientific (2004), and references therein.

[3] ATLAS Technical Design Report, ATLAS TDR 14, CERN/LHCC 99-14, 1999; CMS Physics Technical Design Report, CERN/LHCC 06-021, CMS TDR 8.2, 2006.

[4] H. Baer et al., Phys. Rev. D 75, 095010 (2007).

[5] S. Dawson, E. Eichten and C. Quigg, Phys. Rev. D 31, 1581 (1985).

[6] W. Beenakker et al., Phys. Rev. Lett. 74, 2905 (1995); Z. Phys. C 69, 163 (1995);

W. Beenakker et al., Nucl. Phys. B 515, 3 (1998).

[7] W. Beenakker et al., Nucl. Phys. B 492, 51 (1997).

[8] J. Botts and G. Sterman, Nucl. Phys. B 325, 62 (1989).

[9] N. Kidonakis and G. Sterman, Phys. Lett. B 387 (1996) 867; Nucl. Phys. B 505, 321 (1997).

[10] N. Kidonakis, G. Oderda and G. Sterman, Nucl. Phys. B 525, 299 (1998).

[11] N. Kidonakis, G. Oderda and G. Sterman, Nucl. Phys. B 531, 365 (1998).

[12] R. Bonciani et al., Phys. Lett. B 575, 268 (2003).

[13] R. Bonciani et al., Nucl. Phys. B 529, 424 (1998).

[14] S. Mert Aybat, L. J. Dixon and G. Sterman, Phys. Rev. Lett. 97, 072001 (2006); Phys. Rev. D 74, 074004 (2006).

[15] S. Moch and P. Uwer, arXiv:0804.1476 [hep-ph].

[16] A. Kulesza and L. Motyka, in preparation.

[17] S. Catani et al., Phys. Lett. B 378, 329 (1996).

[18] J. Kodaira and L. Trentadue, Phys. Lett. B 112, 66 (1982).

[19] S. Catani, E. D'Emilio and L. Trentadue, Phys. Lett. B 211, 335 (1988). 
[20] S. Catani et al., Nucl. Phys. B 478, 273 (1996).

[21] W. Beenakker et al., arXiv:hep-ph/9611232; http://www.ph.ed.ac.uk/ tplehn/prospino/

[22] W. K. Tung et al., JHEP 0702, 053 (2007).

[23] L. J. Dixon, L. Magnea and G. Sterman, arXiv:0805.3515 [hep-ph]. 